\title{
B-FACTORY COMMISSIONING AND FIRST RESULT
}

\author{
Shin-ichi Kurokawa, Accelerator Laboratory \\ KEK, High Energy Accelerator Research Organization, Tsukuba, Japan \\ shin-ichi.kurokawa@kek.jp
}

\begin{abstract}
Performances of two B-Factories, PEP-II at SLAC and $\mathrm{KEKB}$ at KEK, have been steadily improved during these two years from the start of physics experiments in mid 1999. PEP-II peak and integrated delivered luminosities per day, per week, and per month $\left(3.30 \times 10^{33} \mathrm{~cm}^{-2} \mathrm{~s}^{-1}\right.$, $184 / \mathrm{pb}, 1036 / \mathrm{pb}, 3.8 / \mathrm{fb}$ ) had surpassed its design goals in October 2000. The performance of KEKB has been improved rapidly in 2001 and it recorded the peak luminosity of $4.04 \times 10^{33} \mathrm{~cm}^{-2} \mathrm{~s}^{-1}$ in June 2001. The maximum integrated logged luminosity per day and per month by BELLE amounted to $224 / \mathrm{pb}$ and $4.7 / \mathrm{fb}$. These high performances of the B-Factories are attributed to successful fine tuning of the machines and also to the suppression of beam blow-up due to electron cloud instabilities by solenoid field imposed on the positron ring vacuum chambers. We reasonably expect that by the end of $2002 \mathrm{BaBar}$ at PEP-II and BELLE at KEKB will have logged 100/fb. A path to a luminosity of $2 \times 10^{34} \mathrm{~cm}^{-2} \mathrm{~s}^{-1}$ at PEP-II has been proposed and a study of super KEKB with a luminosity of $10^{35} \mathrm{~cm}^{-2} \mathrm{~s}^{-1}$ has started. In February 2001, BaBar and Belle have published their first results of CP-Violation.
\end{abstract}

\section{INTRODUCTION AND FEATURES OF PEP-II AND KEKB}

PEP-II at SLAC[1] and KEKB at KEK[2] are highluminosity, two-ring, asymmetric-energy, electronpositron colliders for B-physics. PEP-II rings are housed in a $2.2-\mathrm{km}$ tunnel and a $3.1-\mathrm{GeV}$ positron ring (LER) is installed on top of a 9-GeV electron ring (HER). Electrons and positrons collide at an interaction point (IP) head-on, and dipole magnets near the IP separate them. The overall layout of PEP-II is shown in Fig.1. BaBar detector[3] surrounds the IP

KEKB consists of an 8-GeV electron-ring (HER) and a 3.5-GeV positron ring (LER). HER and LER are housed in a 3-km tunnel and installed side-by-side. Two rings change their inner and outer position in the tunnel twice, once at the IP, and the other at a cross-over point just opposite to the IP. BELLE detector[4] surrounds the IP, where electrons and positrons collide at a finite angle of $\pm 11 \mathrm{mrad}$; whereas, at the cross-over point, two rings have different heights to avoid collisions. Figure 2 shows the layout of KEKB. Table 1 summarizes design parameters of PEP-II and KEKB.

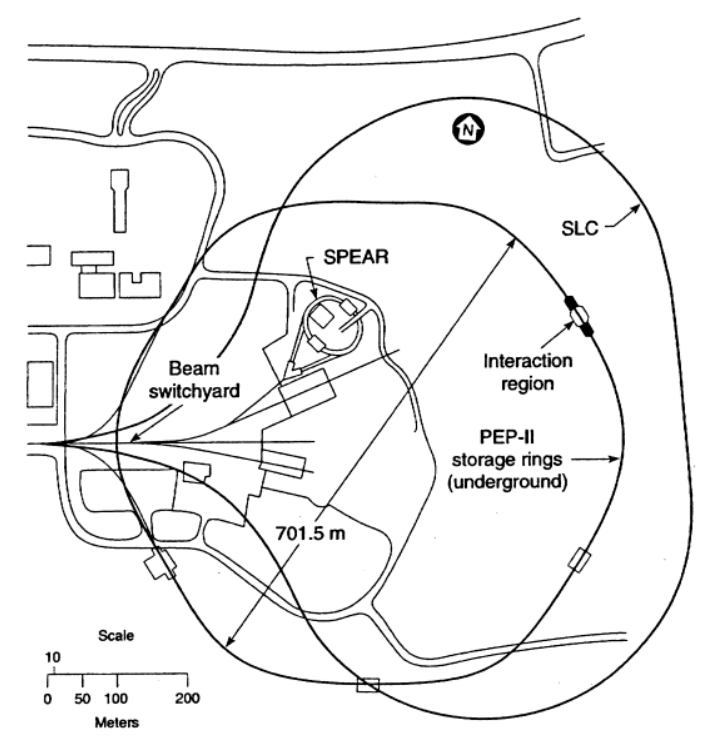

Fig. 1: Schematic layout of PEP-II

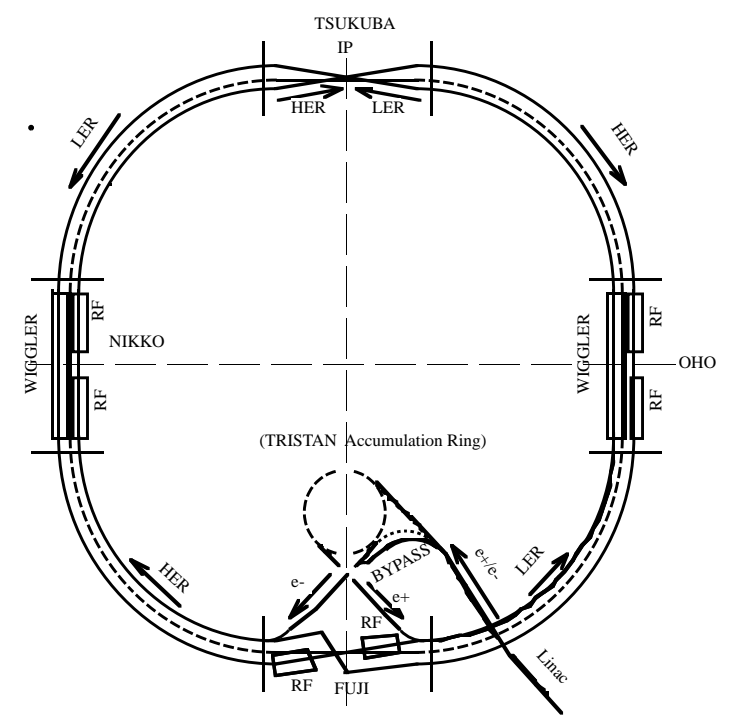

Fig. 2: Schematic layout of KEKB 
Table 1: Main design parameters of PEP-II and KEKB

\begin{tabular}{|l|l|l|}
\hline & PEP-II & KEKB \\
\hline Energy $E / E_{+}(\mathrm{GeV})$ & $9.0 / 3.1$ & $8.0 / 3.5$ \\
\hline Circumference $(\mathrm{m})$ & 2199 & 3016 \\
\hline Luminosity $\left(10^{33} \mathrm{~cm}^{-2} \mathrm{~s}^{-1}\right)$ & 3 & 10 \\
\hline $\begin{array}{l}\text { Beam-beam parameter } \\
\xi_{x} / \xi_{y}\end{array}$ & $0.03 / 0.03$ & $0.039 / 0.052$ \\
\hline$\beta_{x}^{*} / \beta_{y}^{*}$ at IP $(\mathrm{cm})$ & $50 / 1.5$ & $33 / 1.0$ \\
\hline$\sigma_{x}^{*} / \sigma_{y}^{*}$ at IP $(\mu \mathrm{m})$ & $157 / 4.7$ & $90 / 1.9$ \\
\hline Stored current $I / I_{+}$ & $0.75 / 2.15$ & $1.1 / 2.6$ \\
\hline Number of bunches & 1658 & 5000 \\
\hline Bunch spacing $(\mathrm{cm})$ & 1.26 & 59 \\
\hline \begin{tabular}{l} 
Crossing angle \\
\hline
\end{tabular} & 0 & $\pm 11 \mathrm{mrad}$ \\
\hline
\end{tabular}

The luminosity $L$ of an electron-positron collider is given by the following formula:

$$
L=2.2 \times 10^{34} \xi_{y}(1+r) E I / \beta_{y}^{*}
$$

where $\xi_{y}$ is the beam-beam parameter, $r$ the ratio between the vertical beam size $\left(\sigma_{y}^{*}\right)$ to the horizontal beam size $\left(\sigma_{x}^{*}\right)$ at the IP (usually $r<<1$ and is negligible), $I$ the current in ampere, $E$ the beam energy in $\mathrm{GeV}$, and $\beta_{y}^{*}$ the $\beta$-value at the IP in $\mathrm{cm}$. This formula is applicable to both electrons and positrons. In order to reach a high luminosity, $\xi_{y}$ and $I$ should be maximized, and $\beta_{y}^{*}$ minimized. PEP-II design currents are $2.15 \mathrm{~A}$ in LER and $0.75 \mathrm{~A}$ in HER to reach $3.0 \times 10^{33} \mathrm{~cm}^{-2} \mathrm{~s}^{-1}$ with $\xi_{y}$ of 0.03 and $\beta_{y}^{*}$ of $1.5 \mathrm{~cm}$. In KEKB, in order to reach the design luminosity of $1.0 \times 10^{34} \mathrm{~cm}^{-2} \mathrm{~s}^{-1}$, although challenging values such as $\beta_{y}^{*}$ of $1 \mathrm{~cm}$ and $\xi_{y}$ of 0.052 are assumed, 2.6 $\mathrm{A}$ in LER and 1.1 A in HER should be stored. These high stored currents are achieved by distributing them into a large number of bunches, 1658 in PEP-II and 5000 in KEKB.

One of the features of B-Factories is the use of higherorder-modes free accelerating cavities to prevent coupledbunch instabilities. Heavily damped, single-cell, singlemode normal conducting cavities are used at PEP-II[5], while at KEKB both normal conducting cavities called ARES[6] and superconducting cavities[7] are used. ARES consists of three cells: an accelerating cell is connected to an energy-storage cell via a coupling cell. The stored energy of the system is increased by one-order of magnitude. On the other hand, due to its high gradient, a superconducting cavity has a large stored energy by itself. These large stored energies of cavities effectively suppress the coupled-bunch instabilities due to the fundamental mode of cavities[8]. At PEP-II, the coupledbunch instabilities due to the fundamental mode of cavities are damped by using RF feedback with comb filters[9].

KEKB adopts a finite-angle crossing of $\pm 11 \mathrm{mr}$ at IP. Although simulation shows no degradation of luminosity due to the finite-angle crossing, crab-crossing scheme is a fall-back option[10]. In crab crossing, each beam is kicked side-wise by a crab cavity before the IP, collide head-on at the IP, and kicked-back to its original orientation by another crab cavity. Superconducting crab cavities are being developed[11].

Final focus quads should be located under a detector solenoid; the use of iron magnets is precluded. PEP-II uses permanent magnet interaction region quads and dipoles[12], whereas, KEKB uses a pair of superconducting final focus quads, in front of each of which a superconducting anti-solenoid magnet is located and housed in the same cryostat[13].

Both PEP-II and KEKB use copper vacuum chambers for HER, while the type of HER vacuum chambers is different: PEP-II adopts Aluminum antechambers in its LER arc sections, while KEKB uses round copper vacuum chambers.

\section{STATUS OF COMMISSIONING}

\subsection{PEP-II}

HER of PEP-II was completed in June 1997 and LER in July 1998. After commissioning without detector, BaBar was rolled on in May 1999 and physics experiments commenced.

The luminosity of PEP-II increased steadily: on September 14, 1999, it exceeded $1 \times 10^{33} \mathrm{~cm}^{-2} \mathrm{~s}^{-1}$ and reached $1.35 \times 10^{33} \mathrm{~cm}^{-2} \mathrm{~s}^{-1}$, and in April 2000, the luminosity reached $2 \times 10^{33} \mathrm{~cm}^{-2} \mathrm{~s}^{-1}$. During the most of the year 2000 summer run, the beam current in HER was administratively limited to ease stress on a failing vacuum joint in the HER high-power dump. For the last three weeks of the run in October 2000, the HER current was slowly raised and the luminosity followed. On October 29 it exceeded $3 \times 10^{33} \mathrm{~cm}^{-2} \mathrm{~s}^{-1}$ for the first time and later that day the luminosity reached $3.30 \times 10^{33} \mathrm{~cm}^{-2} \mathrm{~s}^{-1}$.

In the three months winter downtime the third LER RF station was installed to allow the LER current to be raised from 1.8 A to 2.7 A. Two new LER movable collimators have been installed near the IP to improve the BaBar background. A new HER vacuum chambers from 10 to $20 \mathrm{~m}$ upstream of BaBar was installed to improve the vacuum level below 1 nTorr and a new HER high-power dump was installed.

PEP-II resumed its operation on February 3, 2001, and the after a machine start-up and beam-tune-ups, it has recovered the former performance. By June 14, 2001, $\mathrm{BaBar}$ has logged 33.1/fb. Table 2 summarizes parameters of PEP-II at its highest luminosity. Figure 3 shows the monthly peak luminosity of PEP-II, and Fig. 4 accumulation of integrated luminosity by BaBar from the start of experiment. 


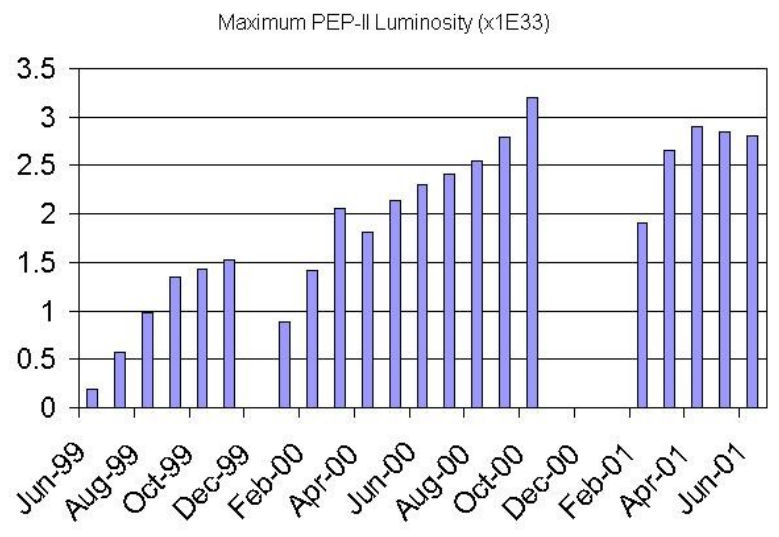

Fig. 3: PEP-II peak monthly luminosity

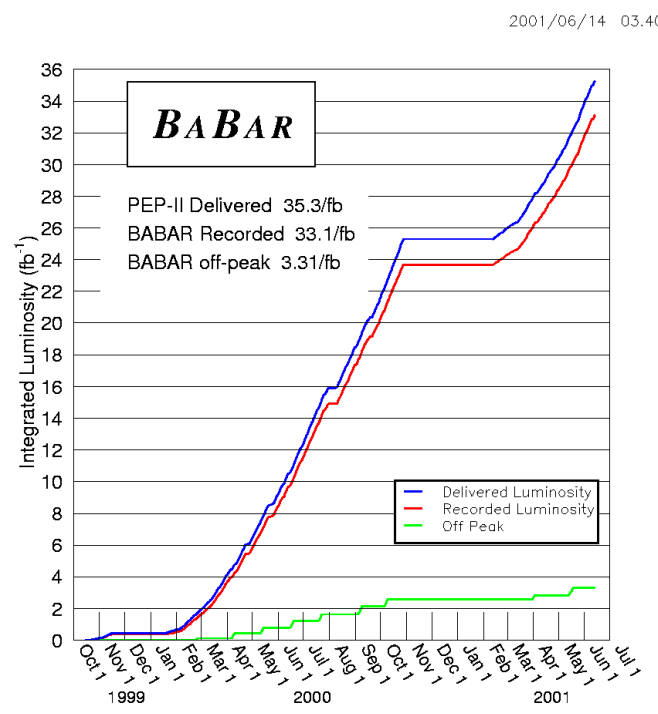

Fig. 4: BaBar accumulated luminosity

Table 2: Parameters of PEP-II at its best performance

\begin{tabular}{|l|c|}
\hline Center of mass energy & 10.28 \\
\hline Crossing angle $(\mathrm{mrad})$ & $<0.1$ \\
\hline Luminosity $\left(10^{33} \mathrm{~cm}^{-2} \mathrm{~s}^{-1}\right)$ & 3.30 \\
\hline Number of bunches & 692 \\
\hline HER current $(\mathrm{A})$ & 0.800 \\
\hline LER currnet $(\mathrm{A})$ & 1.492 \\
\hline HER beam-beam parameters $\xi_{x} / \xi_{y}$ & $0.069 / 0.055$ \\
\hline HER beam-beam parameters $\xi_{x} / \xi_{y}$ & $0.059 / 0.027$ \\
\hline$\beta$ 's at IP (cm) $\beta_{x}{ }^{*} / \beta_{y}^{*}$ & $50 / 1.25$ \\
\hline Horizontal emittance $(\mathrm{nm})$ & $50\left(\mathrm{e}^{-}\right), 30\left(\mathrm{e}^{+}\right)$ \\
\hline Beam size at IP $(\mu \mathrm{m}) \sigma_{x}^{*} / \sigma_{y}^{*}$ & $147 / 5.0$ \\
\hline Injection top-off time $(\mathrm{min})$ & 2 \\
\hline Injection full fill time $(\mathrm{min})$ & 8 \\
\hline
\end{tabular}

\section{$2.2 \mathrm{KEKB}$}

At KEKB both LER and HER were completed simultaneously in November 1998, and after commissioning without detector, BELLE was rolled on to the IP in May 1999 and operation with BELLE started on May 25. By August 5, 1999, a peak luminosity of $2.9 \times$ $10^{32} \mathrm{~cm}^{-2} \mathrm{~s}^{-1}$ was achieved. During the summer shutdown, four ARES cavities were added both in LER and HER.

Operation was resumed on October 12, 1999, and continued until July 23, 2000, with a short break around the New Year Holidays. By July 23, 2000, the peak luminosity increased up to $2.04 \times 10^{33} \mathrm{~cm}^{-2} \mathrm{~s}^{-1}$.

During the summer shutdown of 2000, four superconducting cavities were added to HER in order to increase the current limit up to $900 \mathrm{~mA}$. LER had been equipped with 16 ARES cavities, with which LER could store $2 \mathrm{~A}$. In the operation before the summer shutdown, movable masks of LER suffered from archings, which caused a few cases of vacuum leak. Movable masks of LER, therefore, were replaced with much robust ones. Also during the shutdown, solenoids over $800 \mathrm{~m}$ of LER vacuum ducts were wound.

Operation was resumed on October 10. The increase of the peak luminosity was not prominent in this autumn run; however, the integrated luminosity per day and per week increased considerably due mainly to stable operation of KEKB.

During short winter shutdown around the New Year Holidays, $430 \mathrm{~m}$ of solenoids over LER vacuum ducts were added. The operation was resumed on January 2001, and from that on, the performance of KEKB has been steadily improved. In mid February, vertical tunes of LER and HER were changed from above integer(0.08) to above half integer(0.60). This tune change made the machine much stabler. Also in mid March, the horizontal tunes of both rings were made much closer to half integer from 0.52 to 0.51 , which helped increase the peak luminosity. On March 22, 2001, the peak luminosity surpassed $3.0 \times 10^{33} \mathrm{~cm}^{-2} \mathrm{~s}^{-1}$, and on April 4, 2001, reached $3.31 \times 10^{33} \mathrm{~cm}^{-2} \mathrm{~s}^{-1}$. KEKB had a week-long shutdown in April, during which the HER vertical movable masks were replaced. This allowed the increase of the HER current and the increase of the luminosity followed: on May 2, the luminosity reached $3.84 \times 10^{33} \mathrm{~cm}^{-2} \mathrm{~s}^{-1}$, and on June 13 it exceed $4.0 \times 10^{33} \mathrm{~cm}^{-2} \mathrm{~s}^{-1}$ and reached $4.04 \times$ $10^{33} \mathrm{~cm}^{-2} \mathrm{~s}^{-1}$. Also during this short shutdown, additional $40 \mathrm{~m}$ solenoids were wound. Table 3 shows a parameter list of KEKB when it recorded the highest luminosity of $4.04 \times 10^{33} \mathrm{~cm}^{-2} \mathrm{~s}^{-1}$.

By June 15, 2001, BELLE has logged 28.9/fb. Figure 5 summarizes the history of peak luminosity, integrated logged luminosity per day and accumulated logged luminosity by BELLE.

\subsection{Summary}

PEP-II and KEKB have almost entered into stable running phase from commissioning phase. Both BaBar and BELLE have published their first result of CPviolation measurements in February 2001[14,15]. 
Table 3: Parameters of KEKB at its peak luminosity

\begin{tabular}{|l|c|c|}
\hline & LER & HER \\
\hline Horizontal emittance $(\mathrm{nm})$ & 18 & 24 \\
\hline Beam current $(\mathrm{mA})$ & 885 & 748 \\
\hline Number of bunches & 1154 & 1154 \\
\hline Bunch current $(\mathrm{mA})$ & 0.77 & 0.65 \\
\hline Bunch spacing $(\mathrm{m})$ & \multicolumn{2}{|c|}{2.4} \\
\hline Bunch trains & \multicolumn{2}{|c|}{1} \\
\hline Horizontal size at IP $(\mu \mathrm{m}) \sigma_{x}^{*}$ & 103 & 123 \\
\hline Vertical size at IP $(\mu \mathrm{m}) \sigma_{y}{ }^{*}$ & 2.9 & 2.9 \\
\hline Emittance ratio $(\%)$ & 4.2 & 3.2 \\
\hline$\beta$ `s at IP $(\mathrm{cm}) \beta_{x}{ }^{*} / \beta_{y}^{*}$ & $59 / 0.65$ & $63 / 0.7$ \\
\hline Beam-beam tuneshift $\xi_{x} / \xi_{y}$ & $0.072 / 0.045$ & $0.050 / 0.028$ \\
\hline Beam lifetime $(\mathrm{min})$ & 166 at $854 \mathrm{~mA}$ & 210 at $675 \mathrm{~mA}$ \\
\hline Luminosity $\left(10^{33} \mathrm{~cm}^{-2} \mathrm{~s}^{-1}\right)$ & \multicolumn{2}{|c|}{} \\
\hline
\end{tabular}

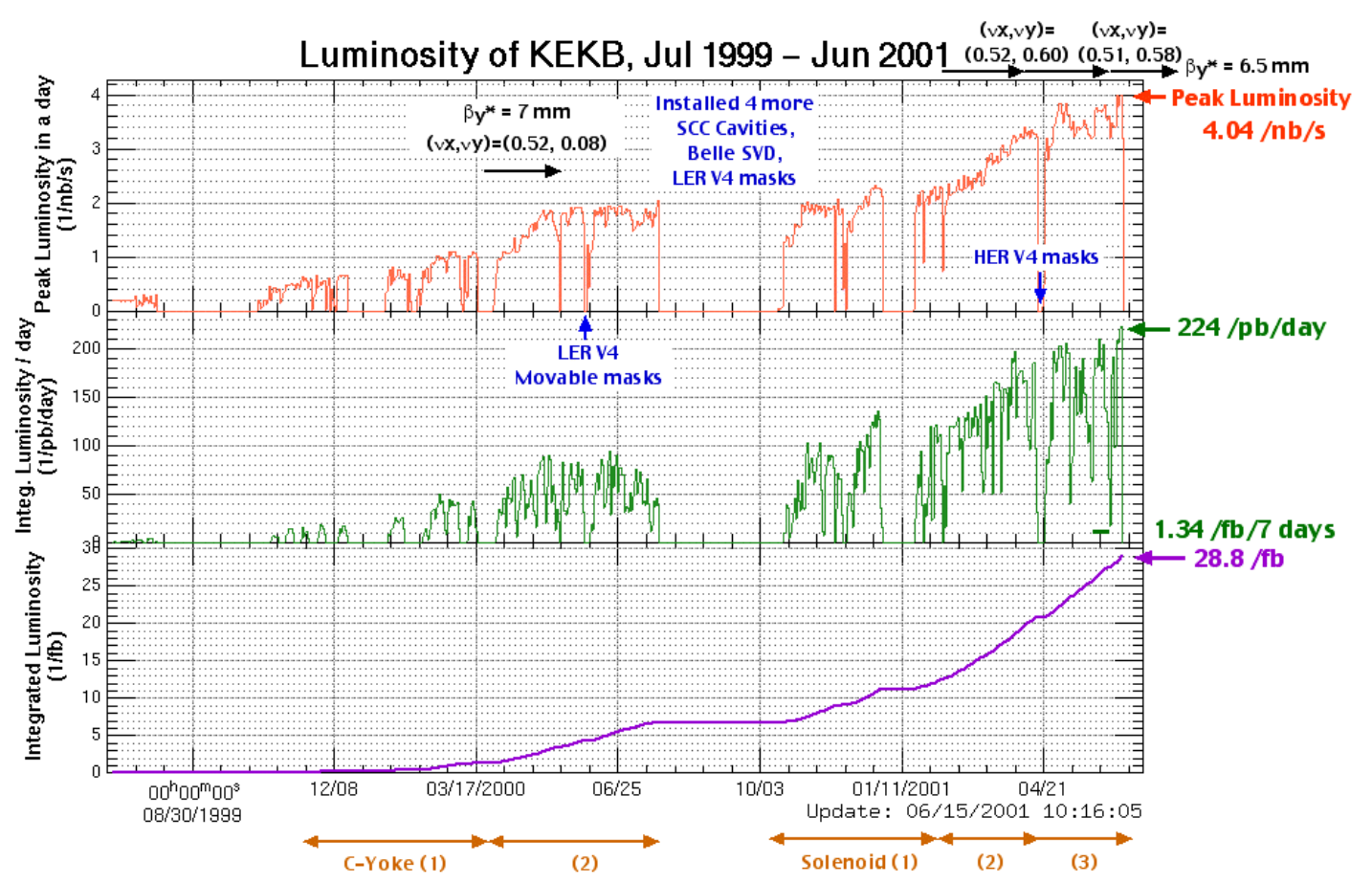

Fig. 5: KEKB performance history

\section{ELECTRON CLOUD INSTABILITIES}

Electron cloud instabilities (ECI) are the most serious instability at PEP-II and KEKB and they limit their performances. Synchrotron light from the positron beam hits the inner wall of vacuum chambers and produces photoelectrons. These photoelectrons are attracted by the beam to form clouds around them. Also electrons created by multipacting form clouds around the beam. The clouds then excite head-tail type oscillations within a bunch and the beam blows up [16]. If we apply solenoid field parallel to the beam, electrons that come out from the inner surface of the vacuum chambers are confined close to the wall. A few 10 gauss solenoid field is strong enough. The use of solenoid field to suppress the ECI instabilities first started at PEP-II (see Fig. 6). They wound solenoids capable of 30 gauss over LER straight section vacuum chambers to suppress multipacitng. A length of $800 \mathrm{~m}$ has been wound resulting a luminosity increase by $70 \%$. With these solenoids the beam size enlargement starts at about $1600 \mathrm{~mA}$. PEP-II is now embarking on a program to wind solenoids in the LER bending arcs to suppress multipacting and photoelectrons there. About 200 chambers each $5 \mathrm{~m}$ long need to be covered.

The solenoid effect to the luminosity was measured at KEKB at the collision mode. Figure 7 shows the result of a study done at KEKB in May, 2001. It shows that (1) when the 430-m long solenoids (NEG-bellows section) wound in January 2001 were turned off, the luminosity 
became smaller by $25 \%$ at high current and the reduction of the luminosity became small when the current decreased, and (2) if all solenoids were turned off the reduction of the luminosity was much larger.

It is highly probable that the rapid improvement of the performance of KEKB observed in year 2001 is mainly due to suppression of beam blow-up by solenoids.

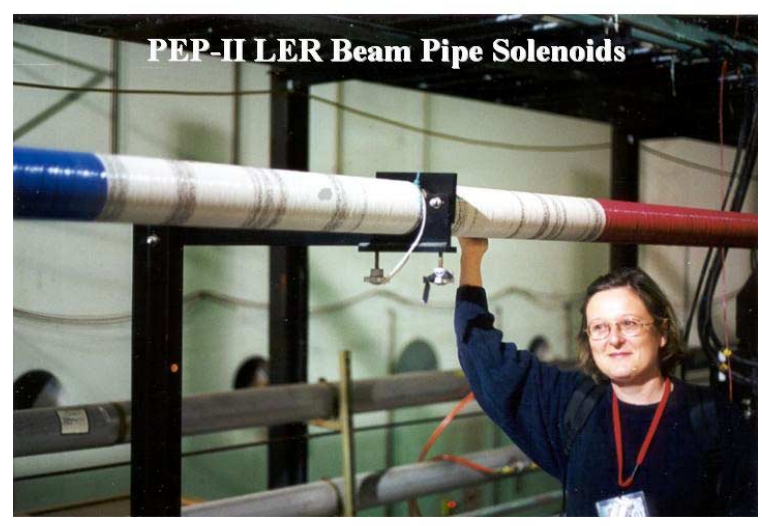

Fig. 6: Solenoids wound over LER straight section vacuum chambers at PEP-II

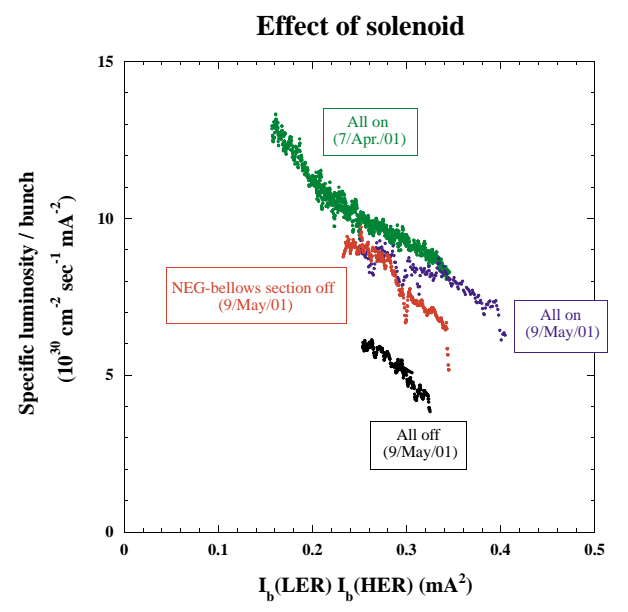

Fig. 7: Specific luminosity per bunch with respect to the product of bunch currents with and without solenoid field.

\section{FUTURE PROSPECTS AND CONCLUSIONS}

Table 4 is a summary of present performances of PEP-II and KEKB. Both B-Factories have a capability of delivering $4-5 / \mathrm{fb} /$ month. It is reasonably expected that by the end of 2002, both BaBar and BELLE will have accumulated $100 / \mathrm{fb}$ and in 3 to 4 years they will accumulate $300 / \mathrm{fb}$. At PEP-II a path has shown to reach $2 \times 10^{34} \mathrm{~cm}^{-2} \mathrm{~s}^{-1}$, whereas at KEKB discussion has started to upgrade it to a super B-Factory with a goal luminosity of $10^{35} \mathrm{~cm}^{-2} \mathrm{~s}^{-1}$.
Table 4: Present performances of PEP-II and KEKB (as of June 14, 2001)

\begin{tabular}{|l|r|r|}
\hline & PEP-II & KEKB \\
\hline Peak luminosity $\left(10^{33} \mathrm{~cm}^{-2} \mathrm{~s}^{-1}\right)$ & 3.30 & 4.04 \\
\hline $\begin{array}{l}\text { Integrated luminosity/shift } \\
(1 / \mathrm{pb})\end{array}$ & $66^{\text {a) }}$ & $79^{\mathrm{b})}$ \\
\hline Integrated luminosity/day (1/pb) & $184^{\text {a) }}$ & $224^{\text {b) }}$ \\
\hline $\begin{array}{l}\text { Integrated luminosity/week } \\
(1 / \text { pb })\end{array}$ & $1036^{\text {a) }}$ & $1336^{\text {b) c) }}$ \\
\hline $\begin{array}{l}\text { Integrated luminosity/month } \\
(1 / \text { fb })\end{array}$ & $3.8^{\text {a) }}$ & $4.7^{\text {b) }}$ \\
\hline $\begin{array}{l}\text { Accumulated luminosity by } \\
\text { detector (1/fb) }\end{array}$ & 33.1 & 28.7 \\
$\begin{array}{l}\text { a) Delivered luminosity. } \\
\text { b) Logged luminosity by detector. } \\
\text { c) Last 7 days }\end{array}$ & \\
\hline
\end{tabular}

\section{ACKNOWLEDGEMENTS}

I thank Dr. John Seeman and Dr. Mike Sullivan of PEPII for their supplying data of PEP-II. I also thank every staff member of KEKB for their efforts to improve the KEKB performance.

\section{REFERENCES}

[1] PEP-II Conceptual Design Report, SLAC Report 418, LBL-PUB-5379, June 1993.

[2] KEKB B-Factory Design Report, KEK Report 95-7, August 1995.

[3] BaBar Technical Design Report, SLAC-R-95-457, March 1995.

[4] A Study of CP Violation in B Meson Decay, KEK Report 95-1, April 1995.

[5] R.A. Rimmer, Phys. Rev. Special Topics-Accelerator and Beams, 3(2000) 102001.

[6] Y. Yamazaki and T. Kageyama, Particle Accel., 44 (1994)107-127.

[7] T. Furuya, et al., Proceedings of $5^{\text {th }}$ European Particle Accelerator Conference (EPAC96), pp.2100-02.

[8] K. Akai, Proceedings of $5^{\text {th }}$ European Particle Accelerator Conference (EPAC96), pp.205-209.

[9] P. Corredoura, Proceedings of 1999 Particle Accelerator Conference (PAC95), pp.435-439.

[10] K. Oide and K. Yokoya, Phys. Rev. A40 (1989) 315.

[11] H. Nakai, et al., Proceedings of Cryogenic Engineering Conference, Montreal, Canada, 1999, Plenum Press, pp.853-860.

[12] M. Sullivan, et al., Proceedings of $5^{\text {th }}$ European Particle Accelerator Conference (EPAC96), pp.460463.

[13] K. Tsuchiya, "Superconducting Final Focusing System for KEKB", in these proceedings.

[14] B. Aubert, et al., Phys. Rev. Lett. 86 (2001) 2515.

[15] A. Abashian, et al., Phys. Rev. Lett. 86 (2001) 2509.

[16] K. Ohmi and F. Zimmermann, Phys. Rev. Lett. 85 3812 (2000). 